\title{
Crackling noise in three-point bending of heterogeneous materials
}

\author{
Gábor Timár and Ferenc Kun* \\ Department of Theoretical Physics, University of Debrecen, \\ P.O. Box 5, H-4010 Debrecen, Hungary \\ (Received 22 December 2010; published 22 April 2011)
}

\begin{abstract}
We study the crackling noise emerging during single crack propagation in a specimen under three-point bending conditions. Computer simulations are carried out in the framework of a discrete element model where the specimen is discretized in terms of convex polygons and cohesive elements are represented by beams. Computer simulations revealed that fracture proceeds in bursts whose size and waiting-time distributions have a power-law functional form with an exponential cutoff. Controlling the degree of brittleness of the sample by the amount of disorder, we obtain a scaling form for the characteristic quantities of crackling noise of quasibrittle materials. Analyzing the spatial structure of damage we show that ahead of the crack tip a process zone is formed as a random sequence of broken and intact mesoscopic elements. We characterize the statistics of the shrinking and expanding steps of the process zone and determine the damage profile in the vicinity of the crack tip.
\end{abstract}

DOI: 10.1103/PhysRevE.83.046115

PACS number(s): 46.50.+a, 89.75.Da, 05.90.+m

\section{INTRODUCTION}

The brittle fracture of materials has two substantially different scenarios depending on the amount of structural disorder: For homogeneous materials such as crystalline solids at the critical stress a single crack is formed that propagates in an unstable manner. However, in materials with a high degree of heterogeneity fracture develops progressively, i.e., under an increasing external load first microcracks nucleate at local weaknesses that may then undergo several steps of growth and arrest. Finally macroscopic fracture occurs as the culmination of the gradual accumulation of damage [1,2]. The nucleation and growth of cracks are accompanied by the emission of elastic waves, which can be recorded in the form of acoustic noise [3]. Measuring acoustic emissions (AEs) on loaded specimens is the primary source of information on the microscopic dynamics of the fracture of heterogeneous brittle materials $[2,4,5]$. During the last two decades a large number of AE experiments were carried out on different materials under quasistatically increasing and constant external loads. These experiments revealed that the energy of acoustic bursts and the waiting times between consecutive events are characterized by power-law distributions. The value of the exponents of crackling noise is found to be characteristic for the type of fracture; i.e., for ductile fracture the exponents are larger than for brittle breaking since large acoustic bursts are suppressed in ductile materials $[5,6]$.

It has long been recognized that in spite of the widely different length scales, AEs of fracturing solids and earthquakes share several features [3]. Recently, it has been pointed out that the probability distribution of waiting times $T$ between consecutive earthquakes can be described by a simple scaling form

$$
P(T) \sim R f(R T),
$$

where $R$ denotes the mean rate of events in the time window considered. The generic law Eq. (1) proved to be valid for all geographical regions unless the time window is sufficiently

*feri@dtp.atomki.hu broad to fulfill the condition of stationarity [7]. Laboratory experiments on the fracture of different types of materials have revealed that the scaling form Eq. (1) is also valid for AEs, and even the scaling function $f(x)$ proved to have the same functional form; i.e., a gamma distribution is obtained $f(x)=$ $A x^{\gamma} \exp (-x / B)$, which means a power-law decay followed by an exponential cutoff [8].

Very recently the statistical features of AEs have been analyzed during three-point bending tests of notched concrete specimens. The notch ensures that the formation of microcracks is dominated by strong spatial correlations in a narrow cross section of the specimen. Varying the detection threshold of acoustic signals for the waiting-time distributions the scaling behavior Eq. (1) was recovered [9,10]. In Ref. [10] the three-point bending experiment was modeled by discretizing the bar in terms of a bundle of fibers. It was a crucial feature of the model that after fiber breakings the load was redistributed equally in the bundle; i.e., spatial correlation of microfractures were not taken into account. Computer simulation of the bending process revealed the same scaling structure [Eq. (1)] of the numerical results; however, for the scaling function $f$ an exponential form was obtained $f \sim \exp (-R T)$. These experimental and theoretical results demonstrate the importance of the range of stress redistribution and correlations of consecutive acoustic events in the statistics of microfractures.

Motivated by these theoretical and experimental findings, in the present paper we investigate the fracture of heterogeneous materials under three-point bending conditions by means of a discrete element model (DEM). Our two-dimensional DEM approach provides a realistic representation of the microstructure of the material, the formation of microcracks, and the emerging complicated stress field naturally accounting for the correlation of microfractures. We analyze both the temporal evolution and spatial structure of damage varying the degree of heterogeneity of the material. Our investigations showed that the fracture proceeds in bursts whose size and waiting-time distributions have power-law behavior with an exponential cutoff. Varying the amount of disorder of the sample we can control the degree of brittleness of the final failure of the material. A scaling form is determined in terms of which the distributions obtained at different amounts of disorder can be collapsed on a master curve. Ahead of the crack tip a process zone is formed composed of broken and 


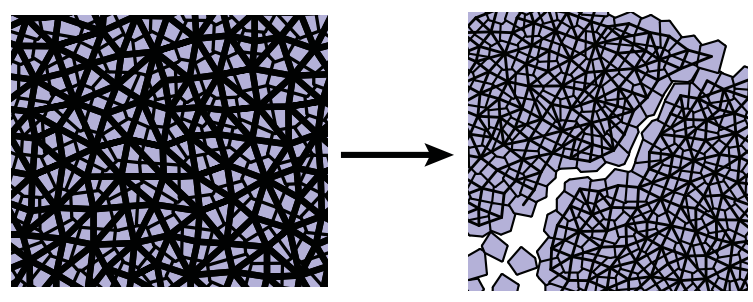

FIG. 1. (Color online) (Left) Neighboring polygons of the initial Voronoi tessellation are connected by beams. This way a triangular beam lattice is obtained. (Right) Due to subsequent breaking of beams a crack forms along the edge of polygons.

intact mesoscopic elements. Our DEM approach allows us to carry out a detailed analysis of the spatial structure of damage as well.

\section{DISCRETE ELEMENT MODEL FOR HETEROGENEOUS MATERIALS}

Three-point bending is a standard engineering test where a bar-shaped specimen is clamped at the two ends and a point load is applied in the middle perpendicular to the longer axis of the bar. Under an increasing load the bar bends and finally breaks due to a crack that appears in the middle along the load direction. This testing method is mainly used in the engineering literature to characterize the quasistatic fracture strength of structural materials such as concrete [11]. On the other hand, three-point bending experiments provide an excellent opportunity to study the propagation of a single crack in a disordered environment, which is a challenging problem for the statistical physics of fracture [2].

Recently, we have worked out a two-dimensional dynamical model of deformable, breakable granular solids, which enables us to perform molecular dynamics simulation of fracture and fragmentation of solids in various experimental situations [12-15]. Our model is an extension of those models that are used to study the behavior of granular materials applying randomly shaped convex polygons to describe grains [12]. The initial set of polygons is obtained by the Voronoi tessellation of a rectangle from which specimens of appropriate shapes can be cut out. The average polygon size $l_{p}$ sets the characteristic length scale of the model system. The polygons are considered to be rigid bodies which can overlap when pressed against each other. We introduce a repulsive force between the overlapping particles proportional to the overlap area [12-15]. To capture the elastic behavior of solids we connect the unbreakable, undeformable polygons (grains) by elastic beams. The beams have two important roles in the model construction: They ensure cohesion, and they are able to break, which is essential to model fracture processes. The beams can be elongated, compressed, sheared, and bent so that they exert forces and torques on the polygons to which they are attached. Figure 1 presents an example of the polygon structure and the beam lattice attached to the polygons.

In the simulations a bar-shaped specimen is considered with longer and shorter side lengths $L$ and $L_{c}$, respectively. In order to make a realistic representation of three-point loading, the three loading plates are realized by additional polygonal elements, i.e., squares in Fig. 2 with side length $S=5 l_{p}$ much

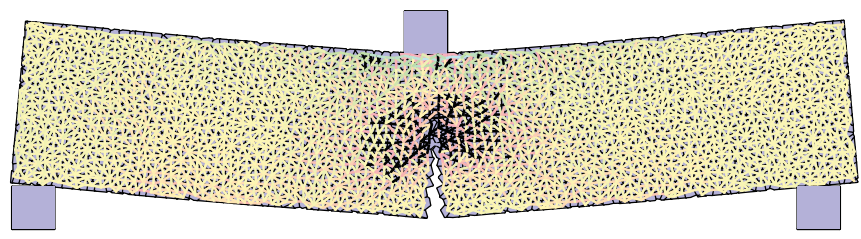

FIG. 2. (Color online) Three-point bending of a bar composed of polygonal particles. The particles are coupled by elastic beams which are colored according to the longitudinal deformation (yellow: nearly unstressed beams; red and black: elongated beams; blue and green: compressed beams). Beams are allowed to break solely along the center line of the bar. A relatively small sample is presented to have a clear view on the details of the model construction. The two loading plates at the bottom are fixed while the third one on the top moves downward.

smaller than the longer side $L=200 l_{p}$ of the bar $S \ll L$. These loading plates interact with the particles of the bar via the overlap force; however, no beams are coupled to them. Strain-controlled loading of the bar is implemented in such a way that the two loading plates at the bottom are fixed while the third one on the top is moved vertically downward in Fig. 2 with a constant speed $v_{0}$. The moving plate overlaps the boundary polygons on the top of the bar, which results in an increasing loading force. The stiffness of the plates is set high enough to keep the overlap below $20 \%$ of the average polygon area. Simulations were carried out varying the value of $v_{0}$ in a range, which allows for an efficient damping of the elastic waves and ensures a reasonable CPU time for the computations. The main advantage of three-point bending tests is that the highly stressed zone, where the crack appears, falls in the middle of the bar, which helps to make efficient monitoring of the fracture process. In order to simplify the numerical measurements on crack propagation, we introduce a "weak" line in the middle of the bar in such a way that solely those beams are allowed to break that connect the two sides of the line (see Fig. 2).

\section{A. Disordered beam breaking}

The beams, modeling cohesive forces between grains, can be broken according to a physical breaking rule, which takes into account the stretching and bending of the connections $[13,14]$

$$
\left(\frac{\varepsilon}{\varepsilon_{\text {th }}}\right)^{2}+\frac{\max \left(\left|\Theta_{1}\right|,\left|\Theta_{2}\right|\right)}{\Theta_{\mathrm{th}}} \geqslant 1 .
$$

Here $\varepsilon$ denotes the longitudinal deformation of a beam, while $\Theta_{1}$ and $\Theta_{2}$ are bending angles at the two beam ends. The breaking rule Eq. (2) contains two parameters $\varepsilon_{\text {th }}, \Theta_{\text {th }}$ controlling the relative importance of the stretching and bending breaking modes, respectively. The energy stored in a beam just before breaking is released in the breakage giving rise to energy dissipation. At the broken beams along the surface of the polygons cracks are generated inside the solid, and as a result of the successive beam breaking the solid falls apart (see Fig. 1). The time evolution of the polygonal solid is obtained by solving the equations of motion of the individual polygons. At each iteration step we evaluate the breaking criterion Eq. (2) and remove those beams that fulfill the condition. The simulation is continued until all beams 
break along the weak interface and the specimen falls apart. (For more details of the model construction see Refs. [12,13].)

The breaking parameters $\varepsilon_{\text {th }}$ and $\Theta_{\text {th }}$ of beams are stochastic variables in the model; i.e. they are sampled from probability density functions $p\left(\varepsilon_{\mathrm{th}}\right)$ and $p\left(\Theta_{\mathrm{th}}\right)$. The Weibull distribution provides a comprehensive description of the stochastic fracture strength of materials; hence, for both threshold values the Weibull form is prescribed:

$$
p_{\lambda, m}(x)=\frac{m}{\lambda}\left(\frac{x}{\lambda}\right)^{m-1} e^{-(x / \lambda)^{m}},
$$

where $x$ denotes the two breaking thresholds $\varepsilon_{\text {th }}, \Theta_{\text {th }}$. The Weibull distribution has two parameters: $\lambda$ sets the characteristic scale of threshold values, while the exponent $m$ determines the scatter of the variable. Increasing the value of the exponent $m$ the width of the Weibull distribution [Eq. (3)] decreases and converges to the delta function in the limit $m \rightarrow \infty$. Varying the scale parameters $\lambda_{\varepsilon}$ and $\lambda_{\Theta}$ of the breaking thresholds the relative importance of stretching and bending can be controlled in the breaking process. For clarity in the present paper we investigate only the two limiting cases of beam breaking dominated by pure stretching or bending with the parameter settings $\lambda_{\varepsilon}=0.05, \lambda_{\Theta}=100$, or $\lambda_{\varepsilon}=100, \lambda_{\Theta}=1$, respectively. The Weibull exponents were changed in the range $1 \leqslant m \leqslant 50$ for both threshold distributions in order to control the amount of disorder in the system. We note that the elastic constants of beam elements depend on the Young's modulus of beams, furthermore, also on their length and cross section. In the model the geometry of beams is determined by the Voronoi tessellation, i.e., the length and cross section of beams are defined as the distance between the center of mass and the length of the common side of the two neighboring polygons, respectively. It has the consequence that besides the strength disorder of beams there is also structural disorder in the system determined by the initial Voronoi tessellation.

\section{MACROSCOPIC RESPONSE}

In our strain controlled three-point bending experiment the mechanical response of the material can be characterized numerically by measuring the force $F$ acting on the moving plate at the top of the sample as a function of time $t$ (see Fig. 2).

Figure 3 presents the force-time curve obtained for a sample with the Weibull exponent $m=2.0$ in the stretching limit. Since the loading plate moves at a constant speed the deflection of the bar is proportional to $t$ so that $F(t)$ can be considered to be the constitutive curve of the sample. It can be seen that the macroscopic response is linear all the way up to the peak, where the force drops suddenly. This drop becomes more and more drastic as we increase the brittleness of the sample by increasing the value of the Weibull exponent. At the beginning of the loading process, the smooth oscillations about the linear in the constitutive curve arise due to elastic waves generated by the loading plate. As the force approaches its maximum, the curve becomes more and more noisy due to internal damage being accumulated in the form of microcracks nucleating throughout the breakable interface. It can be observed in Fig. 3 that after the maximum, the force drops rather drastically; however, the failure is not totally abrupt. The sharp drop-down

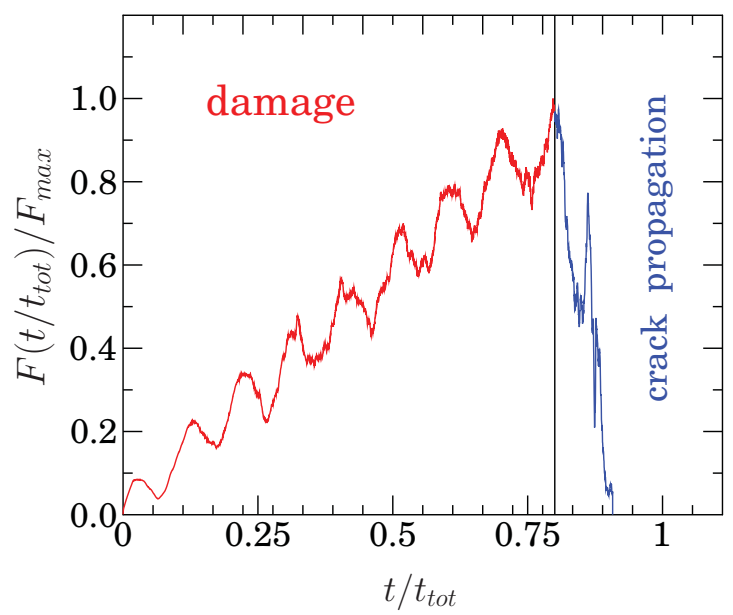

FIG. 3. (Color online) Force $F$ normalized by the maximum value $F_{\max }$ as a function of time $t$ during the loading process. Since the deflection of the bar is proportional to $t$ the curve can be considered as the constitutive curve of the sample. Oscillations occur due to elastic waves generated by the loading process. After the peak the decreasing part of $F(t)$ indicates stable crack propagation where our analysis is focused. $t_{\mathrm{tot}}$ denotes the time of the last beam breaking.

of the force is followed by stable crack propagation where the crack gradually advances until the sample falls apart.

As the bar is loaded at a constant strain rate, microcrackscorresponding to uncorrelated beam breaking events-start nucleating throughout the interface. This way damage is accumulated inside the sample before the onset time of crack propagation. Local beam breaking inside the sample is always brittle; however, the disorder of breaking thresholds can result in a quasibrittle macroscopic response where the constitutive curve exhibits a nonlinear behavior. Due to the disturbing effect of elastic waves, numerically it is difficult to quantify the strength of nonlinearity of the $F(t)$ curve in Fig. 3. Hence, we characterize the degree of brittleness of the sample and its dependence on the amount of threshold disorder by measuring the accumulated damage prior to the peak of the force as a function of the Weibull exponent $m$.

Figure 4 shows the damage parameter $D$ of the model, defined as the fraction of beams broken before a single crack starts propagating, for the stretching and bending limits, as a function of the Weibull exponent $m$. It can be observed that the curves can be very well fitted with the functional form

$$
D(m)=B+A m^{-\mu},
$$

where all parameters $A, B$, and $\mu$ proved to be different for the stretching and bending limits. The power-law form of $D(\mathrm{~m})$ can be motivated by the following simplified assumption: Let us consider a mean-field approximation of the system, where all the beams along the interface share the same $\varepsilon$ strain at any given time during the process. This way the breakable interface of the bar is substituted by a parallel bundle of beams with equal load sharing, whose breaking process can easily be described analytically [16-18]. The fraction of intact beams at any $\varepsilon$ can be given as $1-P(\varepsilon)$, where $P(\varepsilon)$ is the cumulative probability distribution of the breaking thresholds. 


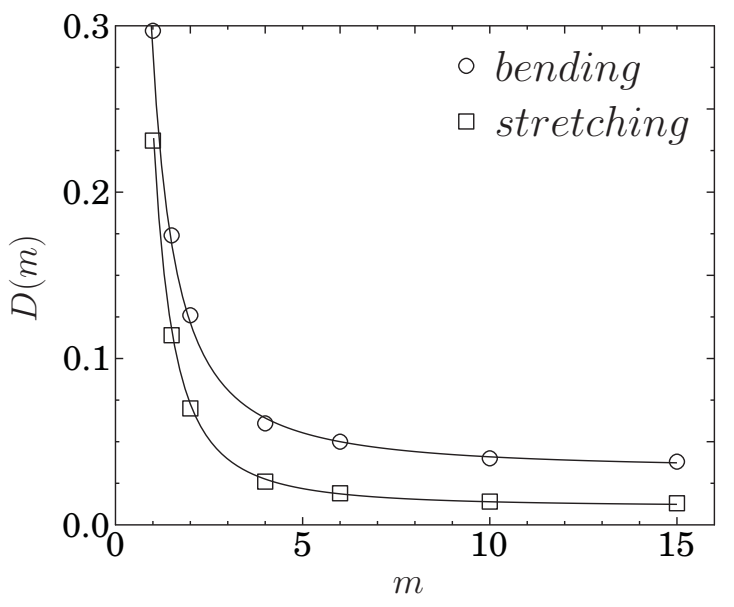

FIG. 4. Damage accumulated up to the peak of $F(t)$ as a function of the Weibull exponent $m$. The curves can be very well fitted with the functional form Eq. (4). The value of the exponent is $\mu=1.9$ and $\mu=1.5$ for the stretching and bending limits, respectively.

The macroscopic stress $\sigma$ as a function of strain $\varepsilon$ can then be written in the form

$$
\sigma(\varepsilon)=[1-P(\varepsilon)] E \varepsilon=e^{-(\varepsilon / \lambda)^{m}} E \varepsilon,
$$

where $E$ is the Young's modulus of the beams. Under strain controlled loading of the bundle, stable crack propagation starts at the peak of the constitutive curve $\sigma(\varepsilon)$. After differentiating Eq. (5), the position of the maximum $\varepsilon_{c}$ reads as $\varepsilon_{c}=\lambda(1 / m)^{1 / m}$ for the Weibull distribution. The fraction of broken beams accumulated up to the peak of $\sigma(\varepsilon)$ can be obtained by plugging $\varepsilon_{c}$ into the cumulative distribution of thresholds $P\left(\varepsilon_{c}\right)$; hence, the damage parameter $D$ as a function of the Weibull exponent $m$ can be cast into the final form for large enough $m$ values

$$
D(m) \approx 1-e^{-(1 / m)} \sim m^{-1} .
$$

The numerical results on the amount of damage prior to the force peak in Fig. 4 are consistent with the above analytic prediction. The higher value of the measured exponents $\mu^{s}=1.9 \pm 0.1$ (stretching) and $\mu^{b}=1.5 \pm 0.1$ (bending) is the consequence of the strain gradient in the load direction, which was completely neglected in the analytic calculations. Note that in the limit of high $m$ values, the amount of damage does not converge to zero; instead it takes a finite value $B>0$. The nonzero value of $B$ in Eq. (4) can be attributed to the structural disorder in the sample, which is present and is the same for all values of the Weibull exponent. This structural disorder gives rise to fluctuations of the beam parameters, which in turn result in a noisy breaking sequence in spite of the constant breaking parameters $[13,14]$.

Perfectly brittle failure of the bar would be characterized by a linear behavior of $F(t)$ up to the maximum without any damaging, which is then followed by an abrupt breaking. Our simulation results demonstrate that varying the amount of threshold disorder we can control the degree of brittleness of the DEM sample from highly (but not perfectly) brittle to quasibrittle. It is a very interesting question how the degree of brittleness affects the properties of crackling noise and the spatial structure of damage along the interface.

\section{CRACKLING NOISE DURING CRACK PROPAGATION}

A snapshot of the computer simulation of a three-point bending test is presented in Fig. 2. The color code shows that the bottom of the specimen is highly elongated, and that is why the crack starts here. In the vicinity of the crack tip the beams are strongly elongated, indicating a high stress concentration ahead of the crack, which provides the driving force for crack propagation. At the top of the bar the color code indicates that compressive stresses arise. The constant speed of the loading plate ensures a strain-controlled loading of the specimen at a fixed strain rate. The low value of the loading speed has the consequence that in each iteration step of the molecular dynamics simulation either no beam breaking occurs or only a single beam breaks. After a local breaking event the stress gets redistributed, increasing the stress concentration on the intact elements ahead of the crack. This load redistribution may give rise to additional breakings, resulting in a correlated trail of breaking events.

In order to identify bursts of local breakings we introduce a correlation time $t_{\text {corr }}$ : If the time difference of two consecutive beam breakings occurring at times $t_{i}$ and $t_{i+1}$ is smaller than the correlation time $t_{i+1}-t_{i}<t_{\text {corr }}$, the two breakings are considered to belong to the same burst. The value of the correlation time was chosen in such a way that it is larger than the time step $\Delta t$ used in the integration of the equation of motion, but it is much smaller than the total duration $t_{\text {tot }}$ of the breaking process; i.e., we set $t_{\text {corr }}=10 \Delta t$ for which $10^{5} t_{\text {corr }}<t_{\text {tot }}$ holds. The size of bursts $\Delta$ is defined as the number of beams breaking during the correlated sequence. When the amount of disorder is very high $m \rightarrow 1$, especially in the bending limit of breakings, it may happen in DEM simulations that very distant beams break within the correlation time, however, without any correlation. To obtain information on the strength of spatial correlations in an avalanche, we calculate the distance $h_{j}=\left|y_{j}-y_{j+1}\right|$ between consecutive beam breakings with the positions $y_{j}$ and $y_{j+1}$ and sum it up inside an avalanche $h=\sum_{j=1}^{\Delta-1} h_{j}$. For a strongly correlated avalanche where each consecutive breaking occurs on adjacent beams the ratio of $h$ and of the burst size $\Delta$ is close to the characteristic polygone size $h / \Delta \approx l_{p}$. In order to filter out avalanches dominated by random coincidences we introduce a threshold value for this ratio; i.e., those avalanches for which $h / \Delta>2 l_{p}$ holds are removed from the statistics. Computer simulations showed that in the stretching limit the above condition has no effect; however, in the bending limit where a high amount of distributed cracking occurs, about $10 \%$ of the avalanches are filtered out due to random coincidences (compare also Fig. 4).

Figure 5 presents the size of bursts in a single fracture simulation at the time of their appearance. It can be seen in the figure that the bursts are separated by silent periods with variable length. These waiting times between bursts characterize the duration of states where the crack tip is pinned due to the presence of some strong beams. At the beginning of the loading process the bursts are small compared to the cross section of the specimen (maximum crack length); however, with increasing deflection of the bar the burst size $\Delta$ increases and reaches a maximum somewhat before the last breaking. After the maximum the burst size decreases, showing that as 


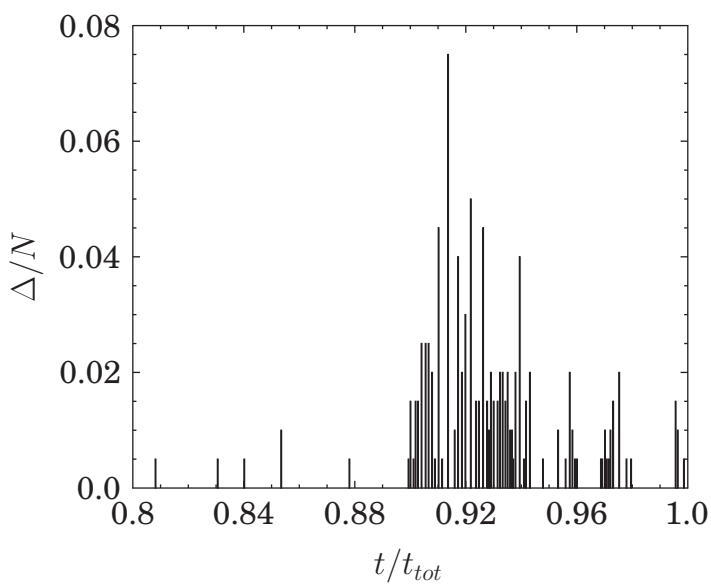

FIG. 5. Time series of bursts in a single fracture simulation. The bursts are correlated breaking sequences of beams, which then result in sudden jumps of the extending crack. $N$ denotes the total number of beams along the weak interface where the crack propagates. For all the simulations its value was set to $N=200$. At the beginning of the loading process, for a considerable time no breaking occurs; most of the breaking events appear at larger deflections beyond the peak of the constitutive curve (see Fig. 3). Hence, we magnify the final section of the bending process.

the crack approaches the top of the bar it slows down due to the compressive zone.

We determined numerically the size distribution of bursts $P(\Delta)$ varying the amount of disorder in the failure thresholds. The size distribution obtained at different values of the Weibull exponent is presented in the insets of Figs. 6 and 7 for the absolute stretching and bending limits, respectively. It is interesting to note that increasing the Weibull exponent $m$, i.e., decreasing the amount of disorder, the bursts get larger, but the functional form does not change. For small bursts a power-law behavior is obtained followed by a rapidly decreasing cutoff regime. The main panels of Figs. 6 and 7 demonstrate that

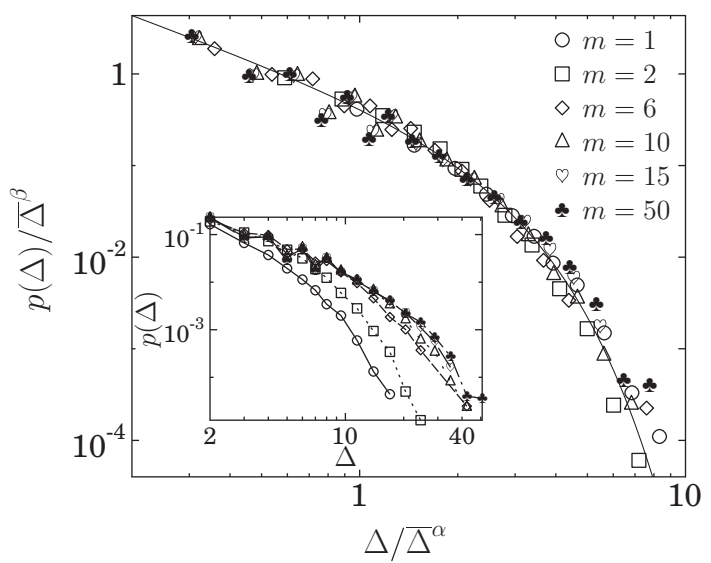

FIG. 6. Inset: Avalanche size distributions for the absolute stretching limit varying the value of the Weibull exponent $m$. The main panel presents the excellent data collapse obtained by rescaling the distributions with the average burst size according to Eq. (7). Scaling exponents: $\alpha_{\Delta}^{s}=1.4 \pm 0.5, \beta_{\Delta}^{s}=1.8 \pm 1$. The parameter values obtained by fitting Eq. (8) are $a_{\Delta}^{s}=0.55, \tau_{\Delta}^{s}=1.3 \pm 0.2, b_{\Delta}^{s}=$ $2.2, \delta_{\Delta}^{s}=1.5 \pm 0.3$.

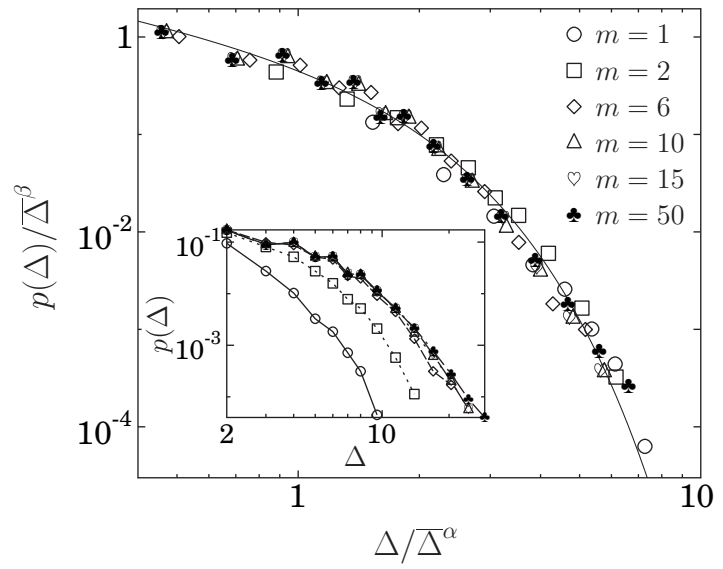

FIG. 7. Inset: avalanche size distributions for the absolute bending limit. The main panel shows that rescaling the distributions according to Eq. (7) an excellent data collapse is obtained. Scaling exponents: $\alpha_{\Delta}^{b}=1.4 \pm 0.5, \beta_{\Delta}^{b}=1.8 \pm 1$. The fit parameters of the scaling function are $a_{\Delta}^{b}=0.85, \tau_{\Delta}^{b}=0.8 \pm 0.3, b_{\Delta}^{b}=1.4, \delta_{\Delta}^{b}=$ $1.3 \pm 0.3$.

using the average burst size $\bar{\Delta}$ as a scaling variable, the burst size distributions $P(\Delta)$ obtained at different $m$ values can be collapsed on a master curve. The data collapse implies the scaling structure

$$
P(\Delta)=\bar{\Delta}^{\beta} f\left(\Delta / \bar{\Delta}^{\alpha}\right),
$$

where the values of the exponents were determined numerically: $\alpha_{\Delta}^{s}=1.4 \pm 0.5, \beta_{\Delta}^{s}=1.8 \pm 1$, and $\alpha_{\Delta}^{b}=1.4 \pm 0.5$, $\beta_{\Delta}^{b}=1.8 \pm 1$, which provide the best quality collapse for stretching and bending, respectively. The scaling function $f$ can be very well fitted by the form

$$
f(x)=a x^{-\tau} e^{-(x / b)^{\delta}},
$$

where the parameter values providing the best fit are $a_{\Delta}^{s}=$ $0.55, \tau_{\Delta}^{s}=1.3 \pm 0.2, b_{\Delta}^{s}=2.2, \delta_{\Delta}^{s}=1.5 \pm 0.3 \quad$ (stretching), and $\quad a_{\Delta}^{b}=0.85, \tau_{\Delta}^{b}=0.8 \pm 0.1, b_{\Delta}^{b}=1.4, \delta_{\Delta}^{b}=1.3 \pm 0.3$ (bending). The results demonstrate that the growth of the crack is not a smooth process; the slow driving results in a jerky crack propagation that is composed of a large number of discrete steps. The growth steps are sudden outbreaks with a variable length. The correlation of consecutive local breakings leads to a power-law functional form limited by an exponential cutoff. The most interesting outcome of the calculations is that the amount of disorder affects only the characteristic scale of bursts, but the functional form and the value of the power-law exponents $\tau_{\Delta}^{s}$ and $\tau_{\Delta}^{b}$ remain the same. We note the differences between the limits of stretching- and bending-dominated breaking; especially the deviation of the exponents $\tau_{\Delta}^{s}$ and $\tau_{\Delta}^{b}$ is beyond the error bars. The functional form Eq. (8) has also been found to provide a good quality description of the amplitude distribution of acoustic bursts in three-point bending experiments on concrete samples $[9,10]$.

It can be observed in Fig. 5 that the bursts are separated by silent periods where no beam breaking occurs. The advancing loading plate gradually increases the load on the system, which reactivates the crack after some waiting time $T$. It can be seen in Fig. 5 that the duration $T$ of these waiting times can vary in a broad range. In Fig. 8 the waiting-time distributions $P(T)$ 


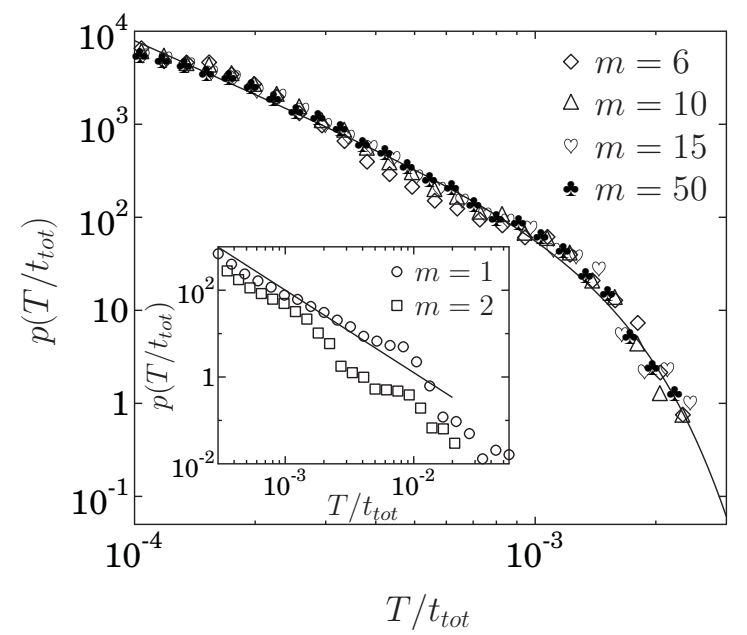

FIG. 8. Waiting-time distributions for absolute stretching. The main panel presents curves for low disorder, where the fit was obtained with the exponent $\tau_{T}^{s}=1.9 \pm 0.15$. The inset shows the corresponding curves for high disorder, where a crossover is obtained to a lower exponent $\tau_{T}^{s}=1.5 \pm 0.1$.

are presented for the stretching limit separated for high (inset) and low disorder (main panel). It is interesting to note that for low enough disorder (main panel of Fig. 8) the distributions $P(T)$ are all the same; no dependence on the Weibull exponent could be pointed out. The functional form of $P(T)$ can be very well fitted by the expression [Eq. (8)] where the value of the exponent $\tau_{T}^{s}=1.9 \pm 0.15$ was obtained. The relatively high value of $\tau_{T}^{s}$ implies that long waiting times are very rare in the trail of bursts when the material is very brittle. However, in the limit of high disorder $m \rightarrow 1$ (see inset of Fig. 8) waiting times span a broader range and reach an order of magnitude larger values than for the very brittle materials with low disorder. The most remarkable feature of waitingtime distributions is that increasing the disorder the exponent of the power-law regime changes to the lower value $\tau_{T}^{s}=$ 1.5 , coinciding with the recurrence time exponent of onedimensional random walks. In the absolute bending limit (see Fig. 9) $P(T)$ has qualitatively the same behavior as in the stretching limit. Due to the fragility of the system at all Weibull exponents $m$, the change of disorder results only in a change of the cutoff; however, the value of the exponent of the power-law regime is constant: $\tau_{T}^{b}=1.8 \pm 0.15$.

\section{SPATIAL STRUCTURE OF DAMAGE}

It has been discussed in Sec. III that at the time of the force drop in Fig. 3, a crack initiates along the breakable interface of the sample and proceeds in a jerky way. The crackling noise analyzed in the previous section characterizes the temporal fluctuations of the advancing crack. A very important advantage of our modeling approach is that it allows us to investigate the spatial structure of damage as well. Based on the sample geometry and loading conditions illustrated in Fig. 2, the crack can be identified as a continuous region of broken beams starting from the bottom of the interface. The high stress concentration ahead of the crack tip and the quenched disorder of the local strength of beams give

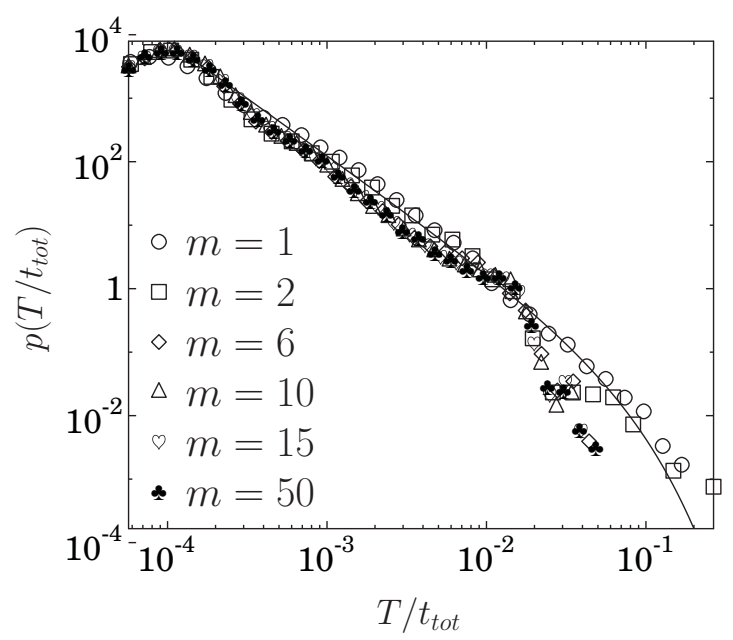

FIG. 9. Waiting time distributions for the absolute bending limit. The amount of disorder affects only the cutoff, but the exponent is constant $\tau_{T}^{b}=1.8 \pm 0.15$.

rise to the emergence of a sequence of broken and intact beams followed by a continuous region of intact elements. Figure 10 illustrates that in the framework of our discrete element model the crack tip can be precisely defined as the position of the first intact beam starting from the bottom of the specimen. The sparse region of broken and intact beams, extending from the crack tip to the last broken fiber, can be identified as the fracture process zone (FPZ) whose dynamics has a strong influence on the time evolution of the breaking process [19]. We note that the extension of the process zone is also affected by the background damage $D$ nucleated in an uncorrelated manner before the crack starts (see Fig. 4). The higher amount of background damage in the bending limit results in a larger extension of the process zone than for the case of stretching-dominated breaking.

\section{A. Dynamics of the process zone}

The dynamics of the process zone strongly determines the advancement of the crack. As the beams break, the extension of the process zone changes in discrete steps: When a new breaking nucleates inside the intact zone the FPZ extends by a length $l_{\text {nucl }}$ called the nucleation length. The process zone

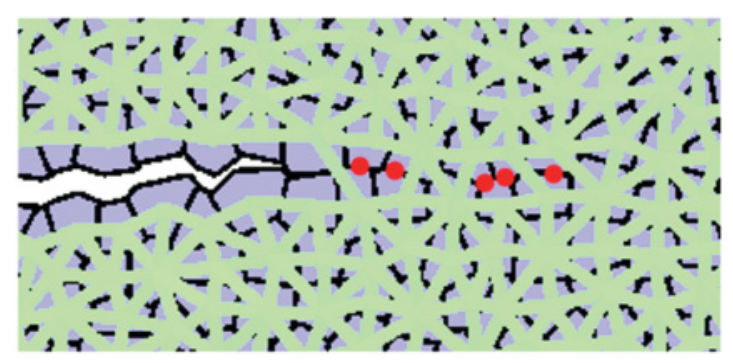

FIG. 10. (Color online) Crack tip and the process zone in front of it. Red dots indicate the positions of broken beams. The process zone is identified as a sequence of broken and intact beams starting at the crack tip and ending at the start of the continuous sequence of intact fibers. 


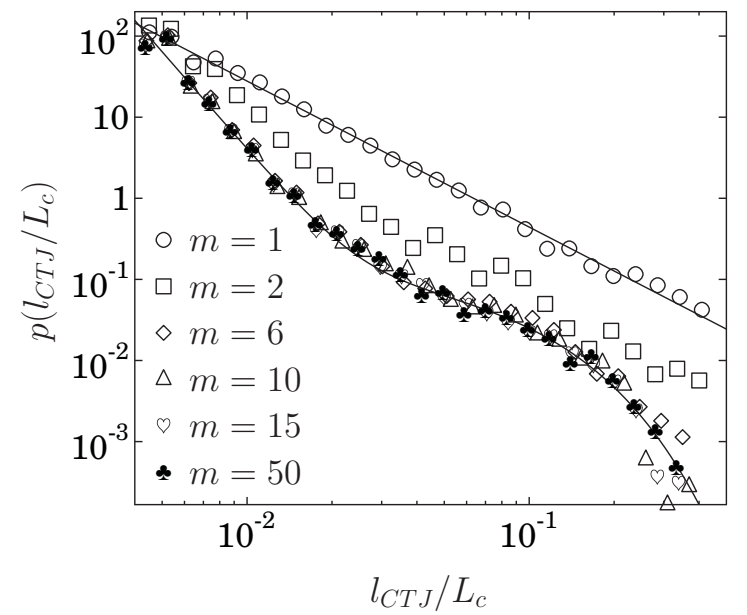

FIG. 11. Crack tip jump length distributions for the absolute bending limit. The values of the exponent $\gamma^{b}$ of the fitted curves are 1.8 and 4.

shrinks when the beam at the crack tip breaks, resulting in a jump of the crack tip (CTJ) with a distance $l_{\text {CTJ }}$.

In order to characterize the dynamics of FPZ we investigate the probability distribution of the nucleation length $p\left(l_{\text {nucl }}\right)$ and of the length of crack tip jumps $p\left(l_{\text {CTJ }}\right)$. We note that the notion of crack tip jump length has also recently been introduced in the framework of Quantized Fracture Mechanics [20-22]. It can be observed in Fig. 11 that for low disorder (high Weibull exponent $m$ ) in the bending limit of breakings the distribution of crack tip jumps has a power-law decay in the regime of small $l_{\text {CTJ }}$ values, which is complemented by an exponential form for large $l_{\text {CTJ }}$

$$
p\left(l_{\mathrm{CTJ}}\right)=a l_{\mathrm{CTJ}}^{-\gamma}+c e^{-l_{\mathrm{CTJ}} / b} .
$$

The additive coupling of the two terms of Eq. (9) shows that the small and large crack tip jumps are generated by different mechanisms; i.e., the small ones are determined by the stress concentration at the crack tip and by the resulting correlation of local breakings. However, the Poisson-like behavior of very large crack tip jumps captured by the second term of the equation originates from the randomness of the initial jump-in of the crack at the onset of crack propagation. The value of the exponent $\gamma^{b}$ changes from $\gamma^{b}=1.8 \pm 0.1$ to $\gamma^{b}=4 \pm 0.2$ as the amount of disorder decreases. In the stretching limit of breakings the sample behaves in a less fragile way, accumulating less background damage before the onset of crack propagation (see also Fig. 4). Hence, in Fig. 12 we obtain a power-law decay with an exponential cutoff but the additive exponential term does not occur. Simulations showed that the value of the exponent does not depend on the amount of disorder $\gamma^{s}=2.2 \pm 0.1$, but it falls between the low- and high-disorder limits of the bending counterpart.

The statistics of crack tip jumps was accumulated over the entire time evolution of the bending process. In order to obtain information about the nucleation length $l_{\text {nucl }}$, we analyze microcracks occurring ahead of the crack tip at the time when the crack spans approximately half of the cross section of the specimen. It can be observed in Fig. 13 that in the bending limit the distribution $p\left(l_{\text {nucl }}\right)$ hardly changes when the amount

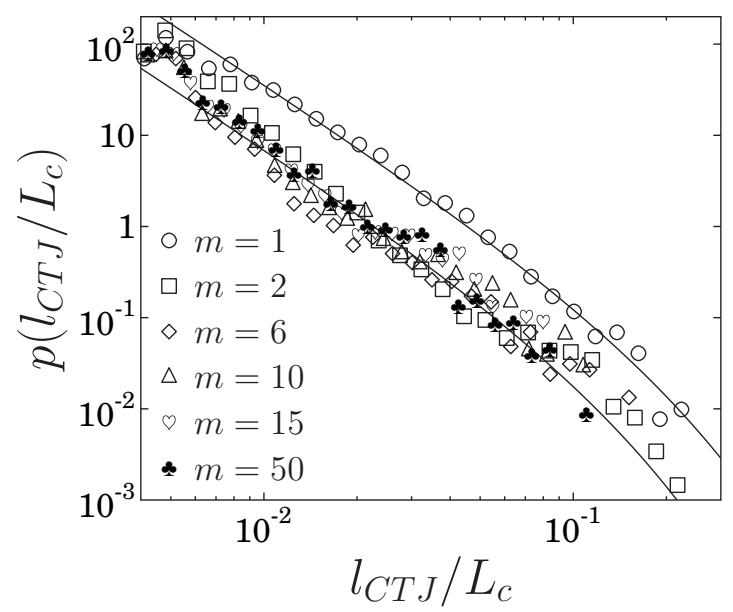

FIG. 12. Crack tip jump length distributions for the absolute stretching limit. The value of the exponent of the power-law regime is $\gamma^{s}=2.2 \pm 0.1$; it does not depend on the amount of disorder.

of disorder is varied. A power-law form is obtained for low length values

$$
p\left(l_{\text {nucl }}\right) \sim l_{\text {nucl }}^{-\kappa}
$$

with an exponent $\kappa^{b}=1.8 \pm 0.15$. When beam breaking is dominated by tensile stresses the situation drastically changes: At high disorder the nucleation distance can extend up to $40 \%$ of the cross section of the specimen as in the bending limit; i.e. very remote beams may also break if they are weak enough (see Fig. 14). At low disorder, however, the nucleation of new broken beams gets localized to the close vicinity of the crack tip where the maximum of $l_{\text {nucl }}$ extends only up to $1 \%-2 \%$ of the cross section. These results on the distance to new nucleations and on the length of crack tip jumps clearly show that in the case of bending-dominated breaking varying the amount of disorder does not have a strong effect on the spatial distribution of damage. The dynamics of the process zone is mainly determined by the long-range redistribution of stresses arising from the bending distorsion. However, when breaking

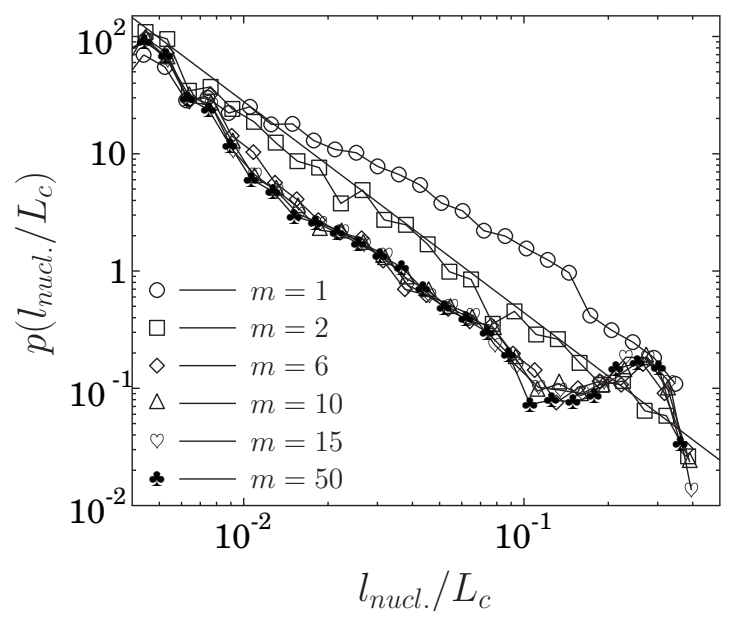

FIG. 13. Nucleation length distributions for the absolute bending limit varying the Weibull exponent $m$. The amount of disorder does not have a relevant effect on the distribution. The exponent of the fitted power law is $\kappa^{b}=1.8$. 


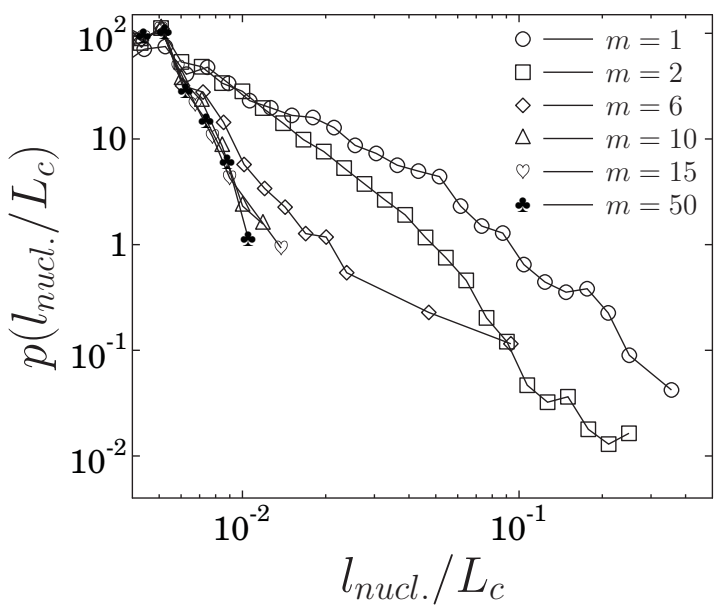

FIG. 14. Nucleation length distributions for the absolute stretching limit. Decreasing disorder leads to localization of damage at the crack tip.

is dominated by tensile deformation, disorder plays a crucial role in the evolution of the fracture process zone; i.e., at low disorder the process zone expands in a large number of small steps while shrinking occurs in the form of a few larger jumps. When the disorder is high, both shrinking and expanding steps can span a broad range.

\section{B. Damage profile}

To obtain quantitative estimates for the extension of the process zone, we calculated the spatial distribution of damage in front of the crack tip when the crack spans half of the specimen's cross section. Figure 15 presents the damage $d$, i.e., the probability of beam breaking as a function of the distance $r$ measured from the crack tip for the stretching limit of the model. The numerical results clearly demonstrate that larger Weibull exponents, i.e., higher degree of brittleness, results in smaller process zones. For all Weibull exponents the curves can be well described by a power-law functional form with an exponential cutoff

$$
d(r) \sim r^{-\rho} \exp \left(r / r_{0}\right)
$$

where the extension of the process zone can be characterized by the length $r_{0}$. It can be observed in Fig. 15 that both the exponent $\rho$ and the characteristic length $r_{0}$ depend on the amount of disorder $m$. Fitting the formula Eq. (11) to the simulated data we obtained the following parameter values: $\rho=0.5, r_{0}=0.05(m=2), \rho=1.0, r_{0}=0.035(m=4)$, $\rho=1.5, r_{0}=0.033(m=6)$.

In order to obtain an analytic understanding of the damage profile $d(r)$ we can start from the result of fracture mechanics that in the vicinity of the crack tip the stress has a power-law decay

$$
\sigma(r)=a r^{-\omega} .
$$

For the exponent $\omega$ linear fracture mechanics predicts the value $\omega=1 / 2$ [19], while fractal cracks and plastic or hyperelastic constitutive laws lead to different values of $\omega$ [23]. Hence,

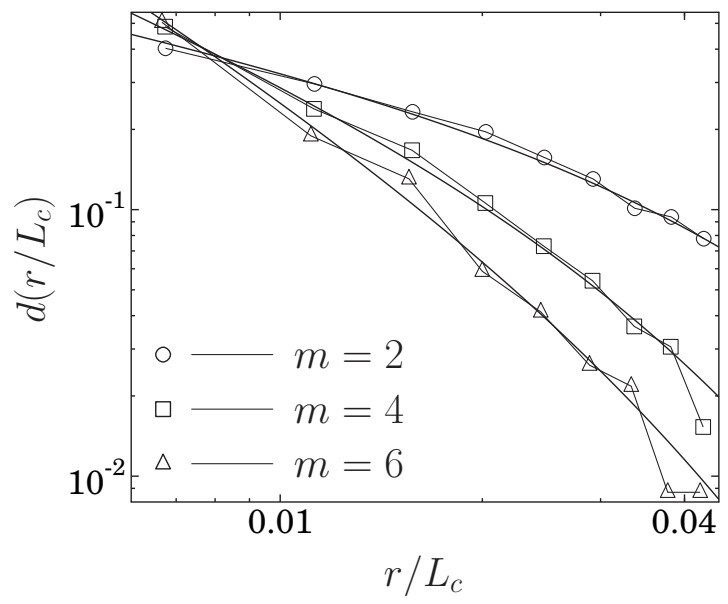

FIG. 15. Damage profile for different values of the Weibull exponent $m$. The distance $r$ measured from the crack tip is normalized by the cross section $L_{c}$ of the specimen. The values of the fitting parameters are $\rho=0.5, r_{0}=0.05(m=2), \rho=1.0, r_{0}=0.035$ $(m=4), \rho=1.5, r_{0}=0.033(m=6)$.

the probability of beam breaking as a function of $r$ can be estimated as

$$
d(r)=P[\sigma(r)],
$$

where $P(x)$ is the cumulative distribution function of the breaking thresholds. Since our breaking thresholds are Weibull distributed, we have $d(r)=1-e^{-(\sigma(r) / \lambda)^{m}}=1-e^{-\left(b r^{-\omega m}\right)}$ where $b=(a / \lambda)^{m}$. Restricting the calculation for small distance we arrive at the form

$$
d(r) \approx b r^{-\omega m} .
$$

The curves of Eq. (11) in Fig. 15 are consistent with the analytical expression Eq. (14) for low values of $r$.

Comparing the results to Eq. (11) the exponent $\rho$ obtained by fitting the numerical data can be written as a product of the exponents of stress decay and disorder $\rho=\omega m$. Substituting the numerical values of $\rho$ and the Weibull exponents $m$ the exponent $\omega$ describing the decay of the stress field can be determined as $\omega \approx 0.25$ for all $m$ values. It is important to emphasize that this value of $\omega$ falls quite close to the analytic result of $\omega=1 / 2$ of linear fracture mechanics [19]; furthermore, the independence of the numerically obtained $\omega$ from the Weibull exponent $m$ shows the consistency of the results. The remarkable feature of the results is that the amount of disorder can have a strong effect on both the shape and extension of the process zone.

\section{DISCUSSION}

The emergence of crackling noise is a ubiquitous phenomenon of the fracture of heterogeneous materials, which can also be exploited to monitor the time evolution of the fracture process. Theoretical studies of crackling noise are usually based on stochastic fracture models such as fiber bundles [9,24,25] and the fuse model [2]. As a novel approach to the problem, in the present paper we investigated the properties of crackling noise emerging during the jerky propagation of a crack in three-point bending tests using a discrete element 
modeling technique. Two limiting cases of breakings were analyzed where stretching or bending dominates the local breaking of beams.

We proposed a numerical technique to identify avalanches based on the temporal and spatial correlation of microfractures.

We showed that for quasibrittle materials the size of bursts and the waiting times between consecutive events are characterized by power-law functional forms with an exponential cutoff. The numerical value of the exponents have a reasonable agreement with recent experimental findings on crackling noise in three-point bending tests on concrete specimens [9,10,26-30]. This agreement also demonstrates the importance of spatial correlations of consecutive microfractures in the emergence of crackling noise.

An important advantage of our DEM approach is that it provides direct access to the spatial structure of damage. Simulations revealed that ahead of the crack tip a process zone develops that is a sparse region of broken and intact elements. The fracture process zone proved to play an important role in the advancement of the crack: On the one hand, the crack progresses by shrinking and expanding steps of the zone; on the other hand, microcracks can shield the stress field around the crack tip, which helps to stabilize the system. Recently the spatial structure of damage has been analyzed in the framework of the fuse model [31,32]. Quasistatic loading simulations were performed starting with a notch in the middle of the fuse lattice analyzing the damage structure in the vicinity of the crack tip just before macroscopic breakdown. It was found that the damage profile has an exponential decay along the line of the crack, and the characteristic length scale of the exponential was suggested as the extension of the process zone. Since linear fracture mechanics predicts a power-law decay of the stress to the background level ahead of the crack tip, the authors argued that the cloud of microcracks shields the crack tip giving rise to an exponential decay. In our system at short distances a power-law decay of the damage profile was obtained which is followed by an exponential cutoff. We think the power-law functional form prevails in our system for the damage profile because microcrack nucleation cannot occur in the two-dimensional plane, but it is restricted to a weak "line" in the sample, which decreases the effect of shielding. This shielding, however, is responsible for the lower exponent of the stress decay $\omega$ and for the exponential cutoff of the damage profile. Computer simulation are complemented by analytic calculations under simplifying conditions, which provided a reasonable agreement with the numerical results.

\section{ACKNOWLEDGMENTS}

The work is supported by OTKA K84157, the Bolyai Janos foundation and TÁMOP 4.2.1-08/1-2008-003 project. The project is implemented through the New Hungary Development Plan, cofinanced by the European Social Fund and the European Regional Development Fund.
[1] A. Johansen and D. Sornette, Eur. Phys. J. B 18, 163 (2000).

[2] M. Alava, P. K. Nukala, and S. Zapperi, Adv. Phys. 55, 349 (2006).

[3] A. Carpinteri and G. Lacidogna (editors), Acoustic Emission and Critical Phenomena from Structural Mechanics to Geophysics (Routledge, New York, 2006).

[4] A. Petri, G. Paparo, A. Vespignani, A. Alippi, and M. Costantini, Phys. Rev. Lett. 73, 3423 (1994).

[5] S. Deschanel, L. Vanel, N. Godin, G. Vigier, and S. Ciliberto, J. Stat. Mech. (2009), P01018.

[6] F. Kun, G. B. Lenkey, N. Takács, and D. L. Beke, Phys. Rev. Lett. 93, 227204 (2004).

[7] A. Corral, Phys. Rev. Lett. 92, 108501 (2004).

[8] J. Davidsen, S. Stanchits, and G. Dresen, Phys. Rev. Lett. 98, 125502 (2007).

[9] G. Niccolini, G. Durin, A. Carpinteri, G. Lacidogna, and A. Manuello, J. Stat. Mech. (2009) P01023.

[10] G. Niccolini, F. Bosia, A. Carpinteri, G. Lacidogna, A. Manuello, and N. Pugno, Phys. Rev. E 80, 026101 (2009).

[11] J. G. M. van Mier, Fracture Processes of Concrete: Assessment of Material Parameters for Fracture Models, New Directions in Civil Engineering (CRC Press, Boca Raton, FL, 1997).

[12] F. Kun and H. J. Herrmann, Comput. Methods Appl. Mech. Eng. 138, 3 (1996).

[13] B. Behera, F. Kun, S. McNamara, and H. J. Herrmann, J. Phys. Condens. Matter 17, 2439 (2005).

[14] G. A. D'Addetta, F. Kun, and E. Ramm, Granular Matter 4, 77 (2002).

[15] F. Kun and H. J. Herrmann, Phys. Rev. E 59, 2623 (1999).
[16] F. Kun, S. Zapperi, and H. J. Herrmann, Eur. Phys. J. B 17, 269 (2000).

[17] R. C. Hidalgo, F. Kun, and H. J. Herrmann, Phys. Rev. E 64, 066122 (2001).

[18] F. Kun and S. Nagy, Phys. Rev. E 77, 016608 (2008).

[19] Z. P. Bazant and J. Planas, Fracture and Size Effect in Concrete and Other Quasibrittle Materials (CRC Press, Boca Raton, FL, 1997).

[20] N. M. Pugno and R. S. Ruoff, Philos. Mag. 84, 2829 (2004).

[21] N. M. Pugno, Int. J. Fract. 140, 159 (2006).

[22] N. M. Pugno, Int. J. Fract. 141, 311 (2006).

[23] N. M. Pugno, A. Carpinteri, M. Ippolito, A. Mattoni, and L. Colombo, Eng. Fract. Mech. 75, 1794 (2008).

[24] R. C. Hidalgo, K. Kovács, I. Pagonabarraga, and F. Kun, Europhys. Lett. 81, 54005 (2008).

[25] R. C. Hidalgo, F. Kun, K. Kovács, and I. Pagonabarraga, Phys. Rev. E 80, 051108 (2009).

[26] N. M. Pugno, J. Phys. Condens. Matter 18, S1971 (2006).

[27] N. M. Pugno, Small 18, S1971 (2008).

[28] F. Bosia, M. J. Buehler, and N. M. Pugno, Phys. Rev. E 82, 056103 (2010).

[29] N. M. Pugno, F. Bosia, and A. Carpinteri, Meas. Sci. Technol. 20, 084028 (2009).

[30] N. M. Pugno and F. Bosia, Nanotechnol. Mag., IEEE 3, 14 (2009).

[31] M. J. Alava, P. K. V. V. Nukala, and S. Zapperi, Phys. Rev. Lett. 100, 055502 (2008).

[32] M. J. Alava, P. K. V. V. Nukala, and S. Zapperi, J. Phys. D: Appl. Phys. 42, 214012 (2009). 